\section{Updates in Neurotology}

\author{
Anirban Biswas ${ }^{1}$ Nilotpal Dutta ${ }^{1}$ \\ ${ }^{1}$ Vertigo and Deafness Clinic, Kolkata, West Bengal, India
}

Ann Otol Neurotol 2019;00:46-50

\section{Repositioning Chair Treatment}

Benign Paroxysmal Positional Vertigo (BPPV) is one of the most common peripheral vestibular disorders and a common cause of vertigo for which patients often visit a vertigo clinic. BPPV can be a very debilitating and traumatic condition. Moreover, several studies have shown a significant relationship between psychological conditions (depression, anxiety) and BPPV. The morbidity related to anxiety and depression can be very easily evaluated with self-assessments tools like Dizziness Handicap Inventory (DHI), the Visual Analog Scale (VAS), and the Hospital Anxiety and Depression Scale (HADS). A study was done by Neil West et al. at Department of Otorhinolaryngology \& Head and Neck Surgery, Rigshospitalet, Copenhagen, Denmark and published in Journal of International Advanced Otology, to evaluate the symptoms of subjective vertigo and associated emotional problems like anxiety \& depression during and after the reposition chair management for refractory BPPV patients. Refractory BPPV is defined as definite or suspected BPPV that did not respond to repeated attempts of manual Canalith Repositioning Maneuver (CRM) treatment. Several limitations in the manual repositioning treatment of BPPV have led to the development of mechanical reposition devices. One of these devices is the TRV chair (TRV Chair; Interacoustics, Denmark; the name 'TRV' is after the inventor/ developer of the chair Thomas Richard-Vitton) which is a mechanical diagnostic and reposition device innovated by him for the management of recurrent BPPV. The advantages of the TRV chair as compared with the conventional way of manual repositioning maneuvers (CRM) are:-1) Improved analytical feasibility 2) Accurate navigation of the head for very precise movement of the contents in the semicircular canals 3) Treat the patients those are unfit for manual treatments like very obese patients or patients with spinal problems4) Allows the examiner to easily rotate the patient 360 degrees along the plane of each semicircular canal(S.C.C) 5) Helps to hold the patient in any position for detailed evaluation of any eye movements (-Fig.1).

The study was performed in a tertiary referral center which is an university medical institution. The patient group was those who were diagnosed to have intractable BPPV
Address for correspondence Anirban Biswas, MBBS, DLO, Vertigo and Deafness Clinic, BJ-252, Salt Lake, Sector-2, Kolkata, 700091, West Bengal, India (e-mail: vertigodeafnesstinnitus@gmail.com).

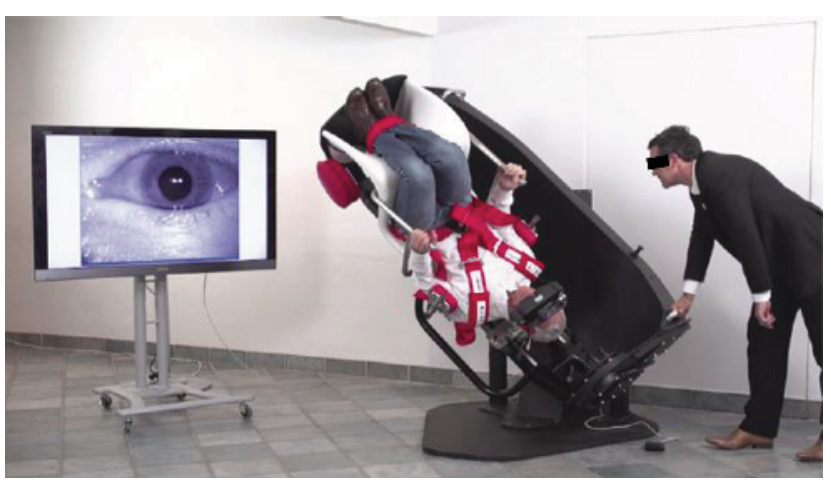

Fig. 1 BPPV treated via the TRV chair. (image courtesy: https://www. interacoustics.com/).

that has failed to conventional manual repositioning (CRM) treatments. Apart from vertigo many patients were also burdened with emotional issues like anxiety, fear and depression. TRV chair offers strict objective control of the head movement in very accurate $3 \mathrm{D}$ rotational planes that can be monitored in the computer while recording the precise eye movement by videonystagmography (VNG). The objective of the study was to evaluate the subjective complaints and emotional issues of the patients with BPPV through questionnaires like VAS, Dizziness Handicapped Index (DHI), and Hospital Anxiety Depression Scale (HADS) after treatment with reposition maneuvers in the TRV chair. The TRV device has a preset $360^{\circ}$ planes for each of the semi-circular canals allowing $360^{\circ}$ movement of the patient along the plane of each semicircular canal in tandem with simultaneous analysis of nystagmus (if any) via the infrared goggles in a computer screen. TRV chair treatment is based on the Epley and the Barbeque (180-270 rotations) maneuvers for posterior and horizontal canal BPPV respectively. Multicanal BPPV is addressed by treating canalolithiasis before cupulolithiasis and repositioning the posterior canal otoliths before the horizontal canal ones. Furthermore, the TRV chair has a potentiated "impact" function, applying decelerating manual forces upon the reposition maneuvers. It was evident from the study that patients with refractory BPPV improved significantly, according to subjective outcomes reported by the
DOI https://doi.org/ 10.1055/s-0039-1698028 ISSN 2581-9607
License terms

() (1) $\ominus \circledast$ 
patients and measurement of the symptoms by the above mentioned questionnaires after the TRV chair management. During the TRV chair management the BPPV was resolved after a mean of two treatment sessions. Thus, the reposition device could significantly reduce disease morbidity burden both physically and psychologically in the group of patients with BPPV who previously failed to respond to conventional manual treatment.

\section{Tumarkin's Crisis and Syncope in Meniere's disease}

Ménière's disease (MD) is characterized by recurrent attacks of heads pinning with fluctuant hearing loss, buzzing or ringing sound in the ear and aural fullness. Apart from the typical symptoms the patients with MD also sometimes complain of difficulties in their gait, postural instability, and a sudden loss of balance which are termed as vestibular drop attacks (VDAs) or Tumarkin attacks. A study done by IImyari Payko et al. at Department of Otolaryngology, University of Tampere, School of Medicine, Tampere, Finland (IP, JZ) confirmed a hypothesis that vestibular drop attacks (VDAs) could cause syncope in patients with Ménière's disease (MD).It was seen that $5 \%$ of patients with MD suffered from syncope, and that it was mostly seen amongst those having VDA. Also the lifetime prevalence of syncope among patients with MD is $12.3 \%$.VDA is defined as a sudden alteration of lower extremity muscle tone without any warning signs or symptoms due to sudden changes in the functions of the otolith organs (Utriculus/saccule) as a result of alterations in pressure gradients inside the inner ear. The mechanism for syncope in VDA was suggested to be due to an impairment in the sympathetic-vestibular reflex that could lead to orthostatic hypotension. Moreover, in the elderly population it was also found that, approximately $2 \%$ of syncope were classified as due to unexplained probably caused by a vestibular disorder. The Syncope jeopardized the quality of life, social interactions, working abilities and induced injuries due to sudden falls. For vestibular syncope, the provoking factors were the frequency of VDA, duration of these attacks, and falls caused by these attacks. The findings also showed that the semicircular canal-derived rotatory vertigo attacks were rare (occurred only in two patients) and did not correlate with syncope and was also supported by animal experiments indicating that the semicircular canal responses do not activate the vestibular sympathetic reflex. However, symptoms such as blurring of vision is a common finding not only in orthostatic intolerance leading to syncope but also in patients with otolith dysfunction similar to that found in present study. Other than the heads pinning the vestibular organ can also trigger complex symptoms, like loss of visual acuity, imbalance, fear of falling, fatigue, cognitive and attention problems. In the present study, patients with syncope also experienced more anxiety disorders. Anxiety disorders are common in patients with MD. In addition to vestibular sympathetic reflex, the link between vestibular disorders and anxiety may be mediated through connections between the superior vestibular nucleus and the parabrachial nucleus, which in turn provides afferents to the limbic system also. Orthostatic reactions in astronauts during immediate post-flight period lead to syncope, and this process and recovery parallels with the recovery of otolith function. Thus the VDA-associated syncope appears to be triggered from the primary otolith afferents that have polarization vectors close to the vertical axis of the head.We assume that due to this diversity of otolith responses, the attacks leading to syncope are originated in specific parts of the otoliths, i.e., parts measuring the vertical axis of the head, whereas otolith ailment in other parts of the vestibulum may not provoke syncope. In some patients with frequent syncope, a continuous heart rate monitoring could provide more information of autonomic dysfunction in VDA. Intratympanic administration of gentamicin have been found to be affective in the treatment of VDA.

\section{Subjective Visual Vertical: an effective Diagnostic tool in Neurotology}

In day to day practice a neurotologist often encounters patients with the symptoms of just unsteadiness and dizziness but no head spinning whatsoever. These symptoms sometimes become so severe and debilitating that they are not able to lead a normal life and their quality of life becomes very poor. One of the causes of unsteadiness and / or dizziness is otolithic dysfunction. The two otolith organs are the UTRICLE and the SACCULE and disorders in these organs is called otolithic dysfunction. The saccular detects vertical acceleration and the utricular detects horizontal acceleration. In otolithic lesions vertical and / or horizontal acceleration i.e., linear acceleration is not adequately sensed and the patient gets a feeling of unsteadiness and dizziness. This is different from typical head spinning which is caused by defects in the semicircular canals which sense angular acceleration in different planes. Not only this, the otolith organs also provide us with the very vital faculty of perception of verticality.

The spatial orientation of the head relative to gravity is constantly monitored by the otolith organs and the information about this is sent to the central nervous system as earth's gravity generates a constant linear acceleration to the body. This information is vital for our survival and it is from this that we can perceive which is up and which is down i.e., whether we are moving up or down and also perceive forward/ backward and side to side movement. In disorders of the balance system, the structural and functional integrity of the otolith systems needs to be tested. Since perception of verticality is one of the functions of the otolith system, we can test otolith function by evaluating the subject's perception of verticality. In a subjective visual vertical test, a person with a normal otolithic system aligns the target very precisely with very little deviation from zero degrees(normal range is $0-2.5$ degrees) as a healthy vestibular system provides equal stimulation to both utricles when the head is straight up, and is instrumental in aligning the target line to the vertical (gravitational vertical) during test and deviation from the actual vertical is $0-2.5$ degrees). However in the acute peripheral vestibular lesions affecting the otolithorgans, the healthy labyrinth correctly detects the stimulation but the 
diseased one provides a deficit which is wrongly interpreted by the brain as a head tilt to the side of the healthy vestibule. The brain most often interprets this discrepancy which is finally interpreted by the patient by projecting a line to a corresponding degree toward the diseased (opposite) labyrinth.

There are various ways by which this perception of verticality (SVV) can be measured. In the past the traditional bucket test and the very cumbersome OVAR Rotational Chair test were used. But refinements in technology have led to development of a very simple, portable diagnostic tool called the subjective visual vertical test (SVV) goggles by which the subject's perception of verticality can be tested.

An article was published in the November 2017 Hearing Review at Tech Topic, by Douglas L. Beck on a newer form of SVV test. The system described consists of a 'Virtual' SVV Goggles for measuring the perception of verticality in a balance disorder patient. It is a more convenient and portable way of measuring the perception of verticality as compared with the other methods. In other methods of SVV portability is a problem as the image has to be cast on a screen by a projector. But this new SVV instrument is portable and the clinician can carry it to different clinics. The Virtual SVV Goggles device includes a light occluding goggle which has a built-in accelerometer to measure head movements and a "candlelike" target to adjust the verticality. The patient has to simply orient and the target inside the goggle using a handheld remote control and any deviation of the set angle of the target line from the tilt angle of the head is measured both in the static and dynamic position of the head. In the static test, the patient's head is at zero degrees of head tilt i.e., the head is perfectly vertical. In the virtual SVV system threshold data of the perception of verticality in a person is presented as in the -Fig. 2. The dark and light green shaded areas represents the normal range.(between the 25th-75th percentiles, and 5th and 95th percentiles respectively).

Thus the patients with a peripheral deficit shows lower level in the tilt to the lesioned side and normal values in tilt positions to the healthy side.

Although there are a lot of tools available to the clinician for detecting any abnormality in the vestibular system like VNG for the lateral canals at low frequency, VHIT for the three SCC at high frequencies, objective tests like cVemp for saccular lesion and oVemp for utricular lesion, but it was seen that some patients have no responses in the o VEMP test due to

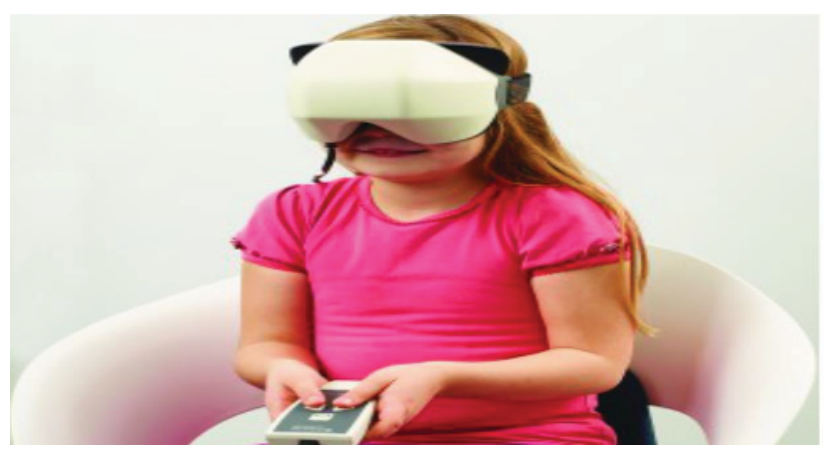

Fig. 2 Child performing a subjective visual vertical test (SVV) pic courtesy: (subjective visual vertical (SVV) and the dizzy patient topic | November 2017 Hearing Review). which the utricular activity and the integrity of the superior vestibular nerve(SVN) cannot be measured, in this patients the role of SVV in the clinical test battery has become very important to diagnose any disorder related to utricular dysfunction and the pathways of the superior vestibular nerve or any abnormality in the cortex of the brain which is responsible for the perception of verticality. Thus SVV has a very important role in the vestibular test battery along with the other tests of vestibular function like the VEMP,VNG,VHIT for diagnosing any abnormality in the vestibular system.

\section{Role of Cognitive Behavioral therapy and Muscle Relaxation Exercises in Tinnitus}

The term tinnitus is derived from the Latin word "tinnire" which means "ringing." It is a phantom phenomenon where the patient has a perception of a sound in the absence of any external source. A large population of people are affected by Tinnitus every year. The US National Institutes of Health and Nutrition have estimated that around $15 \%$ of the general population suffers from chronic Tinnitus. Out of which $\sim 2$ million people face severe impairment in their quality of life due to insomnia and sleep deprivation caused by Tinnitus. A study was conducted by Mojtaba Tavakoli et al at Department of Psychology, Faculty of Education and Psychology, Shahid Chamran University of Ahvaz, Ahvaz, Iran and published in Aud Vestib Res (2019) to find out the effects of cognitive-behavioral training (CBT) and muscle relaxation exercises on the intensity of tinnitus and the quality of sleep in patients with tinnitus. A lot of people suffering from Tinnitus especially women often complain of irritation, sleep related problems and depressive behavior associated with the persistent sound in the ears. Most of these people can easily cope up with these comorbid conditions, however $1.5 \%$ of the population face significant distress and have negative impact in their lives due to these problems associated with tinnitus. The sound in the ears can be of buzzing or ringing type, like a sound of an insect or a gush of wind. Tinnitus can be both subjective and objective in nature, but in most of the people it is subjective. There are several treatment modalities tried for management of Tinnitus like hearing aids, sound therapy, ambient sound enrichment, Tinnitus masking device, benzodiazepines and vasodilators like corticosteroids, but so far no true cure with definitive efficacy has been found for this disease; not of these methods have proved to have a true curative role in the management of the disease. The purpose of these study is to review the efficacy of therapies like Psychological counselling, Cognitive Behavioral Therapy (CBT) and muscle relaxation exercises in the treatment of tinnitus as these methods have the active involvement of the patient suffering from Tinnitus. In these treatment strategies the fundamental concept of the patient regarding tinnitus is completely changed and they start to consider them as nonsignificant entities. The mechanisms utilized by psychological factors to reduce the stress and anxiety caused by tinnitus have been found to be effective in improving quality of sleep. The CBT is a short-term psychotherapy which helps a person to focus on addressing a particular problem such as anxiety, 
insecurity, and phobia. It provides them with the training to identify the maladaptive and disturbing mental problems and to develop a capacity to have a control over negative thoughts generated as a result of these problems. The main pathophysiology behind the effectiveness of CBT in Tinnitus is its profound effect on thoughts, false beliefs, and tinnitus being a psychological perceptive disorder, these techniques helps to modify the inappropriate behaviors of the tinnitus sufferers. The most advantage of these method is that it is very compliant, convenient, safe and without any side effects, hence can be used very effectively to reduce the sufferings of the tinnitus patients.

\section{Conflict of Interest}

None declared. 